\title{
Diffusion-limited binary reactions: the hierarchy of nonclassical regimes for random initial conditions
}

\author{
Panos Argyrakis ${ }^{1}$, Raoul Kopelman \\ Departments of Chemistry and Physics, The University of Michigan, Ann Arbor, MI 48109-1055, USA
}

and

\author{
Katja Lindenberg \\ Department of Chemistry and Institute for Nonlinear Science, University of California at San Diego, \\ La Jolla, CA 92093-0340, USA
}

Received 5 May 1993

\begin{abstract}
We derive a hierarchy of kinetic regimes and crossover conditions for elementary $A+A \rightarrow 0$ and $A+B \rightarrow 0$ batch reactions from a nonclassical reaction-diffusion formalism that includes spatial fluctuations. This paper addresses the case of a spatially random initial distribution of reactants; correlated initial conditions are discussed in another paper. For low dimensions $(d \leqslant 2)$ we find that both $A+A \rightarrow 0$ and $A+B \rightarrow 0$ reactions depart (swiftly) from the classical behavior toward a "depletion zone regime" with non-Hertzian nearest neighbor distributions and nonclassical rate laws. Eventually the $(A-B)$ density difference fluctuations take over in the $A+B \rightarrow 0$ case and lead to the segregated Ovchinnikov-Zeldovich asymptotic behavior with its peculiar rate laws. We give scaling laws for the crossover time and crossover density, with explicit dependencies on the initial density and on the dimension. Similarly, in three dimensions the crossover from the classical to the segregated Zeldovich regime is derived for the $A+B \rightarrow 0$ reaction. Finite size effects differ significantly for the segregated and nonsegregated regimes. In the former case we obtain a relation between aggregate sizes and lattice sizes. Monte Carlo simulations bear out the scaling laws and provide the scaling coefficients.
\end{abstract}

\section{Introduction}

Diffusion-limited binary reactions in low dimensions under appropriate conditions lead to the spontaneous formation of spatial structures and to associated "anomalous" rate laws for the global densities $\rho(t)$ of the reacting species [1-27]. For example, the irreversible reaction $\mathrm{A}+\mathrm{A} \rightarrow 0$ under "normal" circumstances is described by the rate law $\dot{\rho}=-k \rho^{2}$ whereas $[1,4,21-27]$ the asymptotic rate law for dimensions $d<2$ in an infinite volume is $\dot{\rho}=-k \rho^{1+2 / d}$. Physically, the slow-down implied by the higher exponent is a consequence of the spatial distribution of A's. A random or "mixed" distribution of A's has a Hertz distribution [28] of nearest neighbor dis-

1 Permanent address: Department of Physics, University of Thessaloniki, GR-54006 Thessaloniki, Greece. tances and this distribution in turn leads to the normal rate law $[1,15,27]$. The salient characteristic of the Hertz distribution is its maximum at zero separation, indicative of the presence of many extremely close nearest neighbor pairs of reactant particles. An anomalous rate law implies a deviation from the Hertz distribution wherein there are now many fewer close reactant pairs $[1,15,27]$. Indeed, in dimensions lower than two an initially random distribution quickly settles into a distribution that peaks at a finite (nonzero) nearest neighbor separation, leading to an almost crystal-like average arrangement of reactants. This nonrandom distribution arises from the fact that diffusion is not an effective mixing mechanism in low dimensions [29].

Another example of anomalous kinetics in low dimensions is the diffusion-limited irreversible reac- 
tion $A+B \rightarrow 0$. Under normal circumstances the rate laws for the global densities $\rho_{\mathrm{A}}$ and $\rho_{\mathrm{B}}$ are $\dot{\rho}_{\mathrm{A}}=\dot{\rho}_{\mathrm{B}}=$ $-k \rho_{\mathrm{A}} \rho_{\mathrm{B}}$. If $\rho_{\mathrm{A}}(t=0)=\rho_{\mathrm{B}}(t=0) \equiv \rho(t=0)$, then the densities of the two species are equal at all times and we can dispense with the subscripts so that once again $\dot{\rho}=-k \rho^{2}$. The actual asymptotic rate law in an infinite volume in dimensions $d<4$ for an initially random distribution of reactants is instead $\dot{\rho}=$ $-k p^{1+4 / d}$. In this system the principal cause of the anomalous behavior is the formation of aggregates of like particles [1-20]. The spatial regions in which the density of one type of particle is overwhelmingly greater than that of the other grow in time (while of course the total density within each aggregate decreases with time). Since the reaction can essentially only occur at the interfaces between aggregates, and since the number of these interfaces decreases with time, the reaction slows down relative to the rate that would describe a random mixture of reactants. Again, this behavior reflects the fact that diffusion is not an effective mixing mechanism in low dimensions. Initial spatial fluctuations in relative densities can thus grow in size as the reaction that eliminates close opposite pairs proceeds.

This description of the $A+B \rightarrow 0$ reaction depends on the presence of initial spatial fluctuations in the reactant densities. The particular rate law $\dot{\rho}=$ $-k p^{1+4 / d}$ more specifically depends on an initial distribution of reactants that is completely random. A random initial distribution leads to fluctuations in the initial local densities so that the difference in the number of particles of type $A$ and type $B$ in a volume $V$ is initially of the order of the square root of the total number of particles in that volume.

In this paper we discuss the various regimes of kinetic behavior of the densities of reactants from the initial time until the asymptotic behavior is reached. We attempt to characterize the various regimes and to estimate the crossover times from one regime to another. Our analysis in this paper deals with the particular situation when the particles are initially placed in the system in a random way. The parallel analysis for systems with initially correlated pairs of particles is presented elsewhere [30,31]. We also present numerical simulation results in one and two dimensions and analyze these results in terms of our model. Much of our analysis is based on the reaction-dif- fusion model for the local densities $\rho_{\mathrm{A}}(r, t)$ and $\rho_{\mathrm{B}}(r$, t) $[6,31,32]:$

$\dot{\rho}_{\mathrm{A}}(r, t)=D \nabla^{2} \rho_{\mathrm{A}}(r, t)-k_{\ell} \rho_{\mathrm{A}}(r, t) \rho_{\mathrm{B}}(r, t)$

and similarly for $\rho_{\mathrm{B}}(r, t)$, where $k_{\mathrm{l}}$ is the time-independent local rate coefficient and $D$ is the diffusion coefficient for both species. It turns out to be convenient to deal instead with the difference and sum variables $[6,31,32]$

$\gamma(\boldsymbol{r}, t) \equiv \frac{1}{2}\left[\rho_{\mathrm{A}}(\boldsymbol{r}, t)-\rho_{\mathrm{B}}(\boldsymbol{r}, t)\right]$,

$\rho(\boldsymbol{r}, t) \equiv \frac{1}{2}\left[\rho_{\mathrm{A}}(\boldsymbol{r}, t)+\rho_{\mathrm{B}}(\boldsymbol{r}, t)\right]$.

The difference variable satisfies the linear diffusion equation

$\dot{\gamma}(r, t)=D \nabla^{2} \gamma(r, t)$,

which can of course be solved exactly (see below). The sum variable ( 3 ) satisfies the equation obtained by summing (1) and its partner for $\dot{\rho}_{\mathrm{B}}$ :

$\dot{\rho}(\boldsymbol{r}, t)=D \nabla^{2} \rho(\boldsymbol{r}, t)-k_{\mathrm{Q}}\left[\rho^{2}(\boldsymbol{r}, t)-\gamma^{2}(\boldsymbol{r}, t)\right]$.

This equation is more difficult to deal with, and can only be handled approximately.

In section 2 we specify in detail the initial condition to be considered in this paper and explicitly solve for the difference variable for this initial condition. Section 3 describes our simulation methods. Section 4 deals with the analysis of the sum variable equation for the random initial distribution of reactants, the different kinetic regimes that might be observed as the densities decay from their initial distributions, and the crossover times from one sort of kinetic behavior to another. We also present simulation results and analyze them in terms of our theoretical predictions. Section 5 presents our conclusions and a brief overall summary of our results.

\section{Initial condition and difference equation}

Consider the reaction $A+B \rightarrow 0$. We deal with the situation in which A's and B's are initially placed at random locations in the system. Thus, initially $N$ molecules of each type are placed in the system at statistically independent locations $\left\{\boldsymbol{r}_{j}^{\mathbf{A}}\right\}$ and $\left\{\boldsymbol{r}_{j}^{\mathbf{B}}\right\}$ with $j=1, \ldots, N$. The initial local densities are then given by 
$\rho_{\mathrm{A}}(r, 0)=\sum_{j=1}^{N} \delta\left(r-r_{j}^{\mathrm{A}}\right)$

$\rho_{\mathrm{B}}(r, 0)=\sum_{j=1}^{N} \delta\left(\boldsymbol{r}-\boldsymbol{r}_{j}^{\mathrm{B}}\right)$.

The initial distribution is specified by the distribution function

$$
\begin{aligned}
& p\left(\boldsymbol{r}_{1}^{\mathrm{A}}, \ldots, \boldsymbol{r}_{N}^{\mathrm{A}} ; \boldsymbol{r}_{1}^{\mathrm{B}}, \ldots, \boldsymbol{r}_{N}^{\mathrm{B}}\right) \\
& \quad=\prod_{j=1}^{N} p\left(\boldsymbol{r}_{j}^{\mathrm{A}}\right) \prod_{j=1}^{N} p\left(\boldsymbol{r}_{j}^{\mathrm{B}}\right)=\frac{1}{V^{2 N}},
\end{aligned}
$$

where we have used the fact that for a random distribution of molecules $p\left(r^{A}\right)=p\left(r^{B}\right)=1 / V$ and $V$ is the system volume.

\subsection{Solution of difference equations}

The difference variable satisfies the linear diffusion equation (4), which is solved in Appendix A. The quantity of interest involving the difference variable (cf. (5)) is its mean square value $\left\langle\gamma^{2}(r, t)\right\rangle$, where the brackets indicate an average over the initial distribution of reactants. This is also calculated in Appendix A.

The result for a random initial distribution of reactants is given in eq. (A.13):

$$
\left\langle\gamma^{2}(\boldsymbol{r}, t)\right\rangle=\frac{\rho_{0}}{2(8 \pi D t)^{d / 2}} \equiv Q t^{-d / 2} .
$$

Here $\rho_{0}=N / V$ is the initial density of either species and (8) defines the coefficient $Q$.

\subsection{Discretization}

Our Monte Carlo simulations are performed on discrete lattices, as are some of the subsequent detailed calculations. The lattice sites are separated by a lattice constant $a$, and the total number of lattice sites is $L$, so that $V=L a^{d}$. The strict continuum limit corresponds to the limit $a \rightarrow 0$ and $L \rightarrow \infty$ such that $V$ remains finite. The continuum densities $\rho(r, t)$ and $\gamma(r, t)$ have units of number per unit volume, while in our simulations we deal with densities in units of number per lattice site. Denoting the lattice densities by $\rho^{\text {lat }}(l, t)$ where the index $l$ labels the lattice sites, we have the relation $\rho \sim \rho^{\text {lat }} / a^{d}$. The maximum value of $\rho^{\text {lat }}$ when multiple occupancy of a site is not allowed is thus unity. The diffusion coefficient $D$ in the discretized problem has the value $D=\frac{1}{2}$. Thus the distance scale implicit in the diffusion coefficient is different in different dimensions.

In order to simplify notation in our discussion of simulation results in subsequent sections we will in general omit the superscript "lat" even when dealing with lattice densities since the usage will be clear from the context.

\section{Methods of simulations}

The simulations of the $A+B \rightarrow 0$ chemical reaction are performed using the conventional techniques described in our earlier papers [15,33,34]. Briefly, lattices of sizes up to $10^{6}$ sites in one dimension and up to $2000 \times 2000=4 \times 10^{6}$ sites in two dimensions are generated. A and $B$ particles are initially placed in the lattice at random but disallowing multiple occupancy of sites. Cyclic boundary conditions are utilized at the ends of the lattice. The reaction process proceeds in the usual way: The particles diffuse on the lattice by performing independent random walks to nearest neighbor sites. Two A's or two B's are not allowed to occupy the same site. If an A and a B step onto the same site they react, which means that they are removed from the system. Cyclic boundary conditions are employed at the ends of the lattice for the random walk as well. We monitor the particle density as a function of time for times ranging up to $10^{7}$ steps (and in one case up to $10^{8}$ steps).

\section{Kinetic behavior}

Our subsequent analysis is based primarily on the ensemble average of (5) over the initial distribution of reactants:

$$
\begin{aligned}
& \langle\dot{\rho}(r, t)\rangle=D \nabla^{2}\langle\rho(r, t)\rangle \\
& \quad-k_{\ell}\left[\left\langle\rho^{2}(r, t)\right\rangle-\left\langle\gamma^{2}(r, t)\right\rangle\right],
\end{aligned}
$$

which makes the need for (8) apparent. Note that the squared difference variable appears as a source term in the reaction-diffusion equation: the fluctuations in the difference variable thus drive the dynam- 
ics of the system. When the fluctuations in the difference variable are large they play an important role in the overall dynamics of the system.

To proceed from this point one must find a way to deal with the quantity $\left\langle\rho^{2}(r, t)\right\rangle$ and, in particular, with its relation to $\langle\rho(r, t)\rangle$. Rather than attempting to construct a hierarchy of ever higher powers involving $\rho$ and $\gamma$, we proceed along the following lines.

First, for the initially random distribution of reactants we can of course calculate $\left\langle\rho^{2}(r, 0)\right\rangle$ exactly. This calculation is done in Appendix B. From eqs. (B.9) and (B.10) with (B.9) we obtain

$$
\begin{aligned}
& \left\langle\rho^{2}(r, 0)\right\rangle=\langle\rho(r, 0)\rangle^{2}+\rho_{\max }\langle\rho(r, 0)\rangle \\
& =\rho_{0}^{2}+\rho_{\max } \rho_{0} .
\end{aligned}
$$

Here we have dropped terms of $\mathrm{O}\left(N / V^{2}\right)$. In Appendix $B$ we arrived at $(10)$ by starting with a discrete lattice of unit cells of volume $a^{d}$. The quantity $\rho_{\max }$ is the highest possible density of each reactant in the simulations with which our results will subsequently be compared; eq. (10) is obtained if one assumes that at most one molecule can be placed at each lattice site, whence $\rho_{\max }=1 / 2 a^{d}$. In a continuum model strictly speaking $a \rightarrow 0$ and $\rho_{\max } \rightarrow \infty$. However, we retain $a$ as a finite quantity to represent the effect of the finite size of the A and B molecules and the resultant excluded volume effect. Note that the second term on the right-hand side of $(10)$ is greater than the first (unless $\rho_{0}=\rho_{\max }$, which is the maximum possible value of $\rho_{0}$ ).

The entire crux of nonclassical reaction kinetics is the departure of $\left\langle\rho^{2}(r, t)\right\rangle$ from the relation (10). To characterize this departure we write

$$
\left\langle\rho^{2}(r, t)\right\rangle=\eta\langle\rho(r, t)\rangle^{2}+\mu \rho_{\max }\langle\rho(r, t)\rangle,
$$

which constitutes a definition of $\eta$ and $\mu$. We argue below that the variation of $\mu$ between 1 and 0 (and, less importantly, that of $\eta$ between 1 and $1 / 2$ ) captures all the important kinetic regimes of the $A+B \rightarrow 0$ reaction.

\subsection{Kinetic regimes}

The first kinetic regime occurs at $t=0$. The diffusion term in eq. (9) vanishes at $t=0$ since $\langle\rho(r, 0)\rangle$ $=\rho_{0}$ is independent of $r$. The average $\left\langle\rho^{2}(r, 0)\right\rangle$ is given in (10). Clearly, (8) is inappropriate for the evaluation of the initial mean square difference variable (the difficulty arises from the limits that have been taken in evaluating (8)). Instead, we again use (B.9) and (B.10) with (B.9) to evaluate directly

$\left\langle\gamma^{2}(r, 0)\right\rangle=\rho_{\max }\langle\rho(r, 0)\rangle=\rho_{\max } \rho_{0}$

(the infinity in (8) as $t \rightarrow 0$ arises from the fact that in the strict continuum limit $\rho_{\max } \rightarrow \infty$ ). Thus, eqs. (10) and (12) in (9) at $t=0$ yield

$$
\left.\langle\dot{\rho}(r, t)\rangle\right|_{t=0}=-k_{\ell}\langle\rho(r, 0)\rangle^{2} \text {. }
$$

Integrating over $r$ and defining the average global density

$\rho_{\mathrm{g}}(t) \equiv \frac{1}{V} \int \mathrm{d} \boldsymbol{r}\langle\rho(\boldsymbol{r}, t)\rangle$,

we can write

$\left.\dot{\rho}_{\mathrm{g}}(t)\right|_{t=0}=-k_{\ell} \rho_{\mathrm{g}}^{2}(0)$,

Here we have noted that $\rho_{\mathrm{g}}(0)=\langle\rho(r, 0)\rangle=\rho_{0}$. Eq. (15) is precisely the classical rate equation for a bimolecular reaction. We note that the local rate coefficient $k_{\ell}$ is also the rate coefficient for the global rate law in this case. Thus, very near $t=0$ we have $\rho_{\mathrm{g}}(t)=\rho_{0} /\left(1+k_{0} \rho_{0} t\right)$ and also $\langle\rho(r, t)\rangle=\rho_{0} /$ $\left(1+k_{\wp} \rho_{0} t\right)$. We must in general use this more precise form rather than $\langle\rho\rangle \sim t^{-1}$ because $t$ is so short in this regime. Note also that this behavior could have been deduced directly from the original reaction-diffusion equation ( 1 ) since at $t=0$ the diffusion term $D \nabla^{2}\left\langle\rho_{\mathrm{A}}\right\rangle$ vanishes because $\left\langle\rho_{\mathrm{A}}\right\rangle$ is independent of $r$, and the average of the product $\left\langle\rho_{A} \rho_{B}\right\rangle$ separates exactly into the product of the averages,

$$
\left\langle\rho_{\mathrm{A}}(r, 0) \rho_{\mathrm{B}}(r, 0)\right\rangle=\left\langle\rho_{\mathrm{A}}(r, 0)\right\rangle\left\langle\rho_{\mathrm{B}}(r, 0)\right\rangle .
$$

The analysis that leads to classical behavior at very early times is unstable to any fluctuations that lead to a deviation from the strict equality (16). Precisely such fluctuations are of course embodied in the driver $\left\langle\gamma^{2}(r, t)\right\rangle$. Thus, as soon as these fluctuations destroy the equality so that

$$
\left\langle\rho_{\mathrm{A}}(r, t) \rho_{\mathrm{B}}(r, t)\right\rangle \neq\left\langle\rho_{\mathrm{A}}(r, t)\right\rangle\left\langle\rho_{\mathrm{B}}(r, t)\right\rangle,
$$

another kinetic behavior necessarily sets in. This is associated with the nonrandom distribution implied by (17). It is clear that contiguous pairs of $A$ and $B$ molecules will react quickly, and unless diffusion can just as quickly replenish such pairs, there will be de- 
viations from a random distribution as reflected in (17). Although we are unable to estimate the time (which we call $t_{0}$ ) at which the classical behavior ends, simulations (cf. below) indicate that it is extremely short (i.e., while $\rho_{0} t \leqslant 1$ ).

Since (8) is an exact result, any deviations from a strictly random distribution are already built into it. In (11) any deviations from randomness appear in the deviation of $\mu$ and $\eta$ from unity. However, as long as $\mu$ is still of $O(1)$, the second term on the righthand side of (11) dominates the first.

In order to balance contributions of various terms in (9) so as to establish the time dependence of $\langle\rho(r$, $t)\rangle$ in the time regime where the distribution of reactants may no longer be strictly random but $\mu$ is still of $O(1)$ we explicitly substitute (8) and (11) into (9):

$$
\begin{aligned}
& \langle\dot{\rho}(\boldsymbol{r}, t)\rangle=D \nabla^{2}\langle\rho(r, t)\rangle \\
& \quad-k_{\mathrm{l}}\left[\eta\langle\rho(\boldsymbol{r}, t)\rangle^{2}+\mu \rho_{\max }\langle\rho(\boldsymbol{r}, t)\rangle-Q t^{-d / 2}\right] .
\end{aligned}
$$

For $d=1$ the dominant time dependence of $\langle\rho(r$, $t)\rangle$ must be of the form $\langle\rho(r, t)\rangle \sim\left(Q / \mu \rho_{\max }\right) t^{-1 / 2}$. The largest terms in (18), which are the last two terms, then balance each other. All other terms in (18) (including corrections to the dominant behavior) balance each other at higher orders in time. For $d \geqslant 2$ the dominant balance is established with the leading behavior (with perhaps logarithmic corrections in time in two dimensions) $\langle\rho(r, t)\rangle \sim t^{-1}$. When $d=2$ the dominant balance involves the diffusion term, the term linear in the density, and the last term in (18). In dimensions $d \geqslant 3$ the last term becomes unimportant.

In this "intermediate" time regime we have thus found that the main time dependence of the mean density goes as

$$
\begin{array}{rlrl}
\langle\rho(r, t)\rangle & \sim t^{-d / 2}, & d \leqslant 2, \\
& \sim t^{-1}, \quad d>2 .
\end{array}
$$

This behavior is nonclassical in one dimension, and is completely analogous to the nonclassical regime of the $A+A \rightarrow 0$ and $A+A \rightarrow A$ reactions $[15,19,21]$. In those cases the slower-than-classical decay $t^{-1 / 2}$ has been well documented to arise from the "depletion zone" that forms around each surviving reactant after the initially very close pairs have quickly reacted $[1,27]$. Diffusion in one dimension is known to be an ineffective mechanism toward refilling the depletion zone $[15,29,35]$. The same happens here in the $A+B \rightarrow 0$ reaction. After the rapid initial reaction of very nearby A-B pairs (classical regime) each surviving $A$ and $B$ is surrounded by a region relatively poor in the other species $[15,16,38]$. These depletion zones are not efficiently repopulated by diffusion. In our analysis of the dominant balances of terms in (18) in one dimension, the diffusion term was indeed a higher-order contribution while the dominant contribution came from the species fluctuational contribution.

In two dimensions the behavior in time is classical in this regime, $\langle\rho(r, t)\rangle \sim t^{-1}$, perhaps with logarithmic corrections that reflect a remnant of a nonclassical contribution. Such corrections are well documented in the $A+A$ problems [15,34-37]. In dimensions $d \geqslant 3$ the behavior is dominated by the classical contributions. Diffusion is now effective in countering any depletion around reactants, and species density fluctuations play only a higher order role.

After a sufficiently long time has elapsed, it is well known $[1-5,15,16]$ that the system evolves towards macroscopic segregation and that $\langle\rho(r, t)\rangle \sim t^{-d / 4}$ for $d<4$. This behavior is captured by our analysis if we set $\mu=\mathrm{O}\left(\langle\rho\rangle / \rho_{\max }\right)$ and $\eta=1 / 2$ in (11) (see below). The choice $\eta=1 / 2$ implies a complete segregation of species, i.e., that $\left\langle\rho_{\mathrm{A}}(r, t) \rho_{\mathrm{B}}(r, t)\right\rangle=0$. This choice is not crucial to the term balancing arguments. More important is the choice of $\mu$. One might be tempted to assume that the $t^{-d / 4}$ behavior is mainly due to the macroscopic segregation, and that the spatial distribution of molecules within each aggregate is of less importance. This is not the case: indeed, were we to assume an essentially random distribution within each aggregate we would set $\mu \approx 1$ and we would be back to the previous analysis, with the result that $\langle\rho(r, t)\rangle \sim t^{-d / 2}$ for $d \leqslant 2$. In reality the formation of macroscopic aggregates is accompanied by a nonrandom (clumped up) distribution within each aggregate $[15,16]$. Hence the choice $\mu \ll 1$. We will see that setting $\mu=\mathrm{O}\left(\langle\rho\rangle / \rho_{\max }\right)$ captures the proper behavior. Thus in this regime in place of eq. (18) we now analyze the balances implicit in 


$$
\begin{aligned}
& \langle\dot{p}(r, t)\rangle=D \nabla^{2}\langle\rho(r, t)\rangle \\
& \quad-k_{Q}\left[\alpha\langle\rho(r, t)\rangle^{2}-Q t^{-d / 2}\right] .
\end{aligned}
$$

where $\alpha=\mathrm{O}(1)$. This is the form that we assumed in our earlier work [6,31,32].

For $d \leqslant 4$ the dominant balance must occur inside the square brackets, whence

$$
\langle\rho(r, t)\rangle \sim\left(\frac{Q}{\alpha}\right)^{1 / 2} t^{-d / 4} .
$$

The correction to (21) that will allow the balance of the remaining terms has a leading contribution of order $t^{-(1+d / 4)}$. For $d>4$ a balance occurs if $\langle\rho(r$, $t)>\sim B t^{-1}$ (classical behavior) with $B$ independent of $\boldsymbol{r}$. An added correction of order $t^{-2}$ allows the balance of the contributions at the next higher order, which does not involve the $\left\langle\gamma^{2}\right\rangle \sim t^{-d / 2}$ term at all (this latter term does enter when $d \leqslant 4$ ).

Any simulation with which we wish to compare our analysis necessarily takes place in a finite volume. At sufficiently long times, finite volume effects will therefore affect the results of such simulations. It is well known that finite volume effects eventually cause an exponential decay of the density:

$$
\langle\rho(r, t)\rangle \sim \mathrm{e}^{-x t},
$$

where $\chi$ is a constant inversely proportional to the size of the system. This then is the dominant decay at very long times. This behavior is expected to take over when the size of each aggregate is of the order of the size of the system, or (if the initial density is too small to lead to segregation before finite size effects set in ) when the average distance between particles is of the order of the size of the system.

\subsection{Crossovers}

The "major" crossover time $t_{\mathrm{s}}$ and average crossover density $\rho_{\mathrm{s}}$ for $d \leqslant 4$ that we wish to estimate occur when the $t^{-d / 4}$ segregation behavior sets in. For $d \leqslant 2$ this occurs from the depletion zone or residual depletion zone regime while for $d>2$ it occurs directly from the classical regime. In either case, the characteristic that determines this onset is the rough balance of all three terms in the square brackets in (18). We recall that well before this onset $\mu \sim \mathrm{O}(1)$ while in the segregation regime $\mu \sim \mathrm{O}\left(\langle\rho\rangle / \rho_{\max }\right)$. At crossover we set $\eta=1 / 2$ assuming substantial segre- gation to have begun. We write the crossover density as a fraction of the initial density,

$\rho_{\mathrm{s}} \sim f_{d} \rho_{0}$,

where $f_{d}$ is a dimension-dependent fraction assumed to be of $O(1)$ and independent of the initial density. The balance of the first two terms in the square brackets in (18) then yields $\mu_{\mathrm{s}} \sim f_{d} \rho_{0} / 2 \rho_{\max }$. The balance of the first and third terms, $\rho_{s}^{2} / 2 \sim$ $Q t_{s}^{-d / 2} \sim f_{d}^{2} \rho_{0}^{2} / 2$, yields for the crossover time, with $Q$ given by (8)

$t_{\mathrm{s}} \sim \frac{1}{8 \pi D \rho_{0}^{2 / d} f_{d}^{4 / d}}$.

Eqs. (23) and (24) are the main results of this section to be tested against numerical simulations. The value of $f_{d}$ must be determined from the simulations. If our assumption that $f_{d}$ is independent of $\rho_{0}$ is correct, then $t_{\mathrm{s}} \rho_{0}^{2 / d}$ should be independent of the initial density $\rho_{0}$. Likewise, the ratio of the crossover density to the initial density, $\rho_{\mathrm{s}} / \rho_{0}$, should be independent of $\rho_{0}$.

For $d=1$ there is an additional crossover at very early times from the classical $\left[\rho_{0} /\left(1+k_{\mathrm{Q}} t \rho_{0}\right)\right]$ to the depletion zone $t^{-1 / 2}$ behavior. We do not have a quantitative estimate of this crossover time. It may be similar to that for the $A+A \rightarrow A$ reaction, solved exactly by Ben-Avraham and Doering [19].

Finally, at long times the finite size of the system causes an exponential decay of the densities. When the initial density is high enough for there to be a segregation regime, the finite size effects set in when the aggregates of like molecules are of the order of the size of the system. For $d=1$ and $d=2$ it is known $[4,5,38]$ that the linear size of the aggregates on the average grows with time as $a_{d}(D t)^{1 / 2}$. Here $a_{d}$ is a dimension-dependent constant of proportionality which, based on the work of Leyvraz and Redner [38], appears to be approximately 4 in one dimension. There is actually a distribution of aggregates around this average which is skewed toward large aggregates. Finite size effects are thus expected to set in at a time $t_{\mathrm{f}}$ such that the average linear size of the aggregates is some fraction $g_{d}=\mathrm{O}(1)$ of the linear size $L$ of the system, $a_{d}\left(D t_{\mathrm{f}}\right)^{1 / 2} \sim g_{d} L$, i.e.,

$t_{\mathrm{f}} \sim g_{d}^{2} \frac{L^{2}}{a_{d}^{2} D}$ 
The dimension-dependent fraction $g_{d}$ is expected to be independent of the initial density, and must be determined from simulations.

On the other hand, if the initial density is low then segregation may never be observed because finite size effects set in when the average distance between particles is of the order of the size of the system, i.e., when $\rho_{\mathrm{f}}^{-1} \sim L^{d}$. The associated time $t_{\mathrm{f}}$ depends on whether the density $\rho_{\mathrm{f}}$ is achieved in the depletion zone regime or in the classical regime, and must be extracted accordingly.

Fig. 1 summarizes a possible progression of kinetic behaviors as discussed above. In the figure we have assumed that $t_{\mathrm{f}} \gg t_{\mathrm{s}}$.

\subsection{Comparison with Monte Carlo simulations}

Fig. 2 shows our Monte Carlo simulation results for the reactant density as a function of time in onedimensional lattices of various sizes $L$. The initial density in all cases is $\rho_{0}=0.2$ for each species. Here and below densities are always in units of particles per site. The initial slope in all cases is seen to be the same as that of the A+A problem - indeed, the initial slope in fig. 2 overlaps that of a direct A+A simulation. In turn, $A+A$ kinetics is initially classical (slope $=1$ in fig. 2) but very quickly crosses over to the $t^{-1 / 2}$ behavior characteristic of the depletion zone in the $A+A$ reactions (slope $=1 / 2$ in fig. 2 ). We find that the "average slope" over the first 10 steps is 0.55 . At very long times the largest lattices show the Zeldovich behavior. Indeed, for the largest lattice we find an average slope of 0.249 over the last decade of the simulation. The crossover time from the A+A depletion zone behavior to the Zeldovich behavior is discussed below and found to be $t_{\mathrm{s}} \sim 10^{2}$. The smaller lattice simulations show the "peel off" due to finite lattice size effects. Note that each of the simulations for $L=100,1000$, and 10,000 was cut off when only a single particle of each kind was left on the average. For $L=100$ we find that finite size effects begin to set in when $t=t_{\mathrm{f}} \sim 20$. According to eq. (25), the average size of the aggregates is approximately $g_{1} L \sim 4\left(D t_{\mathrm{f}}\right)^{1 / 2} \sim 13$, that is, $g_{1}=0.13$. Thus, each aggregate of each species is on the average larger than $1 / 10$ of the size of the system (which of course must contain at least two aggregates). There is a substantial probability of even larger aggregates [38].

Since we assume that the fraction $g_{d}$ is independent of $L$, we can use the value obtained above, $g_{1} \sim 0.13$, to predict the crossover times $t_{\mathrm{f}}$ for the other lattices. We find for $L=1000$ that $t_{\mathrm{f}} \sim 2 \times 10^{3}$, and for $L=10000$ we obtain $t_{\mathrm{f}} \sim 2 \times 10^{5}$. Both are consistent with the simulation results. To emphasize this point,

(a)

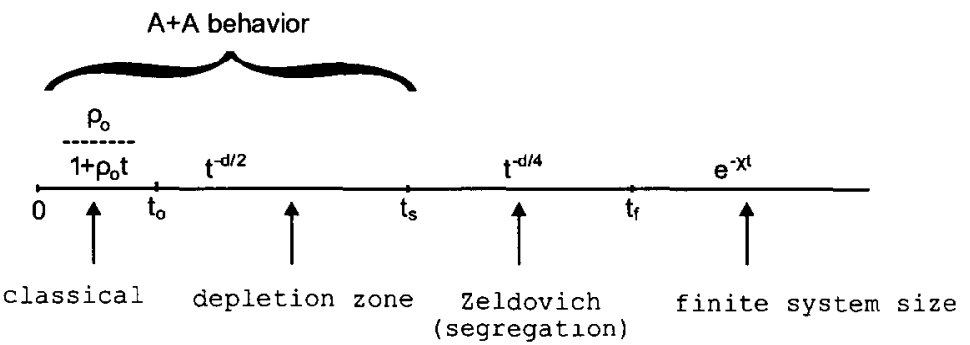

(b)

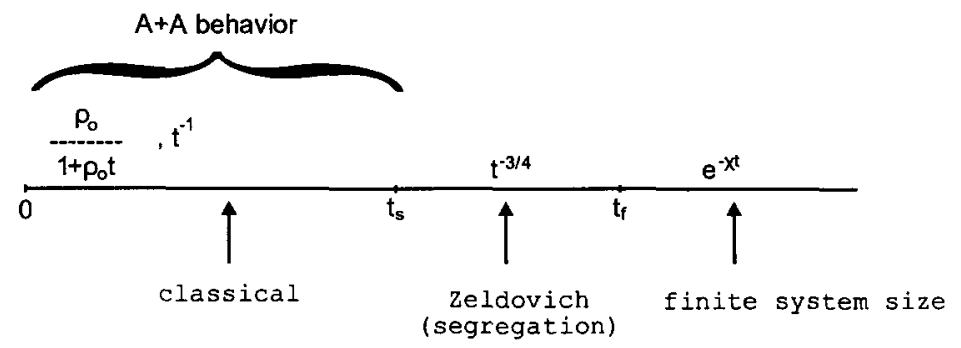

Fig. 1. Possible time progressions of kinetic behaviors discussed in the text for a random initial condition. (a) $d \leqslant 2 ;$ (b) $d=3$. 


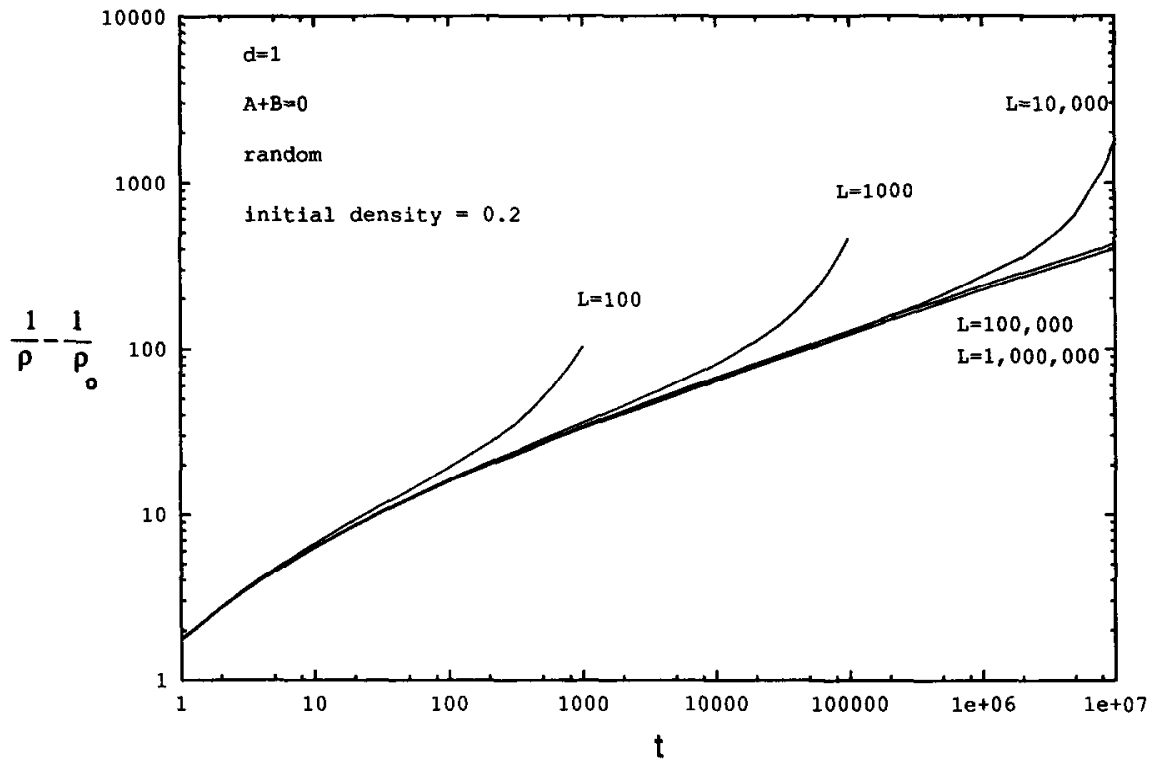

Fig. 2. $\langle\rho\rangle^{-1}-\rho_{0}^{-1}$ versus $t$ for lattices of various sizes $L$ in one dimension with a random initial condition. The initial density of each species in number per lattice site is $\rho_{0}=0.2$ in all cases.

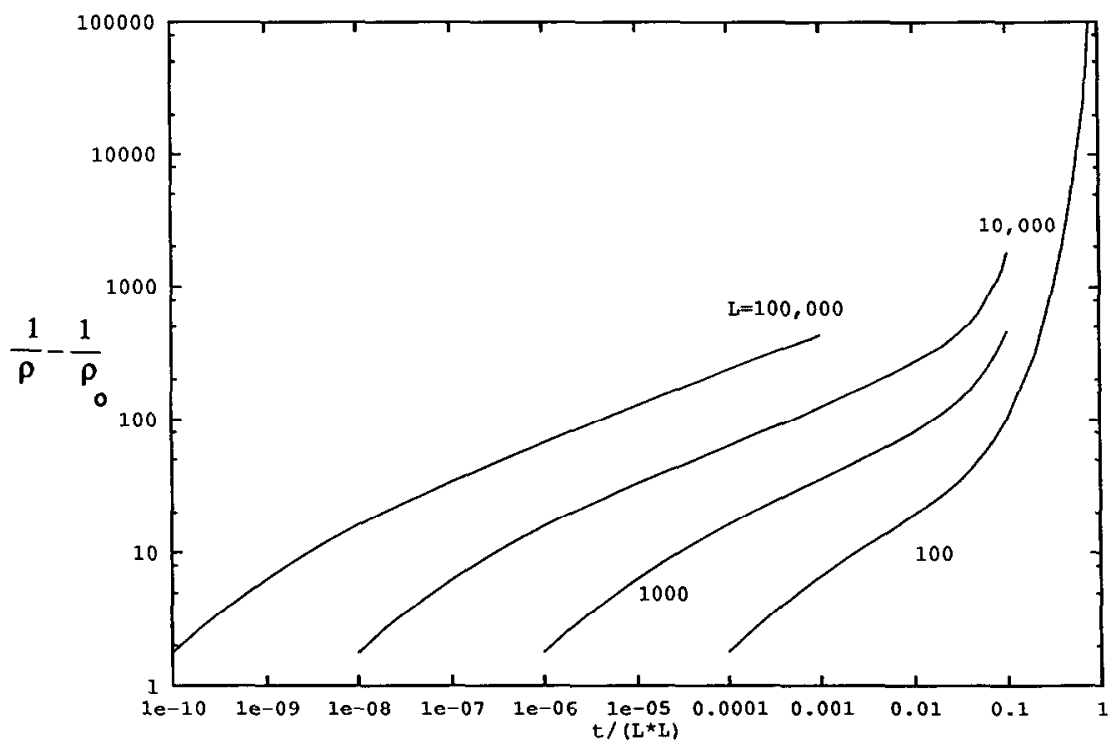

Fig. 3. $\langle\rho\rangle^{-1}-\rho_{0}^{-1}$ versus rescaled time $t / L^{2}$ for the same cases as in fig. 2.

in fig. 3 we have replotted the results of fig. 2 as a function of the scaled time $t / L^{2}$. On this scale the finite size effects for all the finite lattices begin to appear at approximately $t_{\mathrm{f}} / L^{2} \sim 2 \times 10^{-3}$. This figure makes evident the scaling relation $t_{\mathrm{f}} \sim L^{2}$.
Note that although the larger systems of course contain larger aggregates when finite system size effects set in, the number of particles per aggregate at that point is smaller in the larger lattices since more time has gone by and therefore the total density has 
had an opportunity to decrease further than in the smaller lattices.

Fig. 4 shows our Monte Carlo simulation results for the reactant density as a function of scaled time in a one-dimensional lattice for various initial densities $\rho_{0}$. Straight lines have been drawn to aid in the visualization of the crossover times $t_{\mathrm{s}}$ from one kinetic behavior $\left(t^{-1 / 2}\right.$ depletion zone behavior at early times) to another $\left(t^{-1 / 4}\right.$ Zeldovich behavior at later times). The lowest densitics do not reach the crossover within the time regime shown. Finite lattice size effects are not apparent in the time regimes shown in this figure. The crossover time $t_{\mathrm{s}}$ is of course not sharply defined and therefore these lines are not to be taken too literally. The product $t_{\mathrm{s}} \rho_{0}^{2}$ is clearly fairly insensitive to the initial condition, as predicted in (24). The observation that $t_{s} \rho_{0}^{2}=\mathrm{O}\left(10^{0}\right)-\mathrm{O}\left(10^{1}\right)$ leads to $f_{d} \sim 0.3-0.5$. This in turn leads to values of $\rho_{\mathrm{s}} \sim f_{d} \rho_{0}$ that are indeed consistent with the crossover densities in fig. 4.

Fig. 5 shows the Monte Carlo results for the reactant density versus time in two-dimensional lattices of various sizes (compare with fig. 2). Again the initial density in all cases is $\rho_{0}=0.2$ for each species. The initial slope is consistent with the $\mathrm{A}+\mathrm{A}$ behavior of unity within logarithmic corrections. The average slope over the first 10 steps is 0.78 . At long times the largest lattices show Zeldovich behavior: we find a slope of 0.53 in the time domain $10^{4}-10^{5}$ for the largest lattice - the crossover time to this behavior occurs at $t_{\mathrm{s}} \sim 250$ (see below).

For $L=60$ we find that finite size effects begin to set in when $t=t_{\mathrm{f}} \sim 150$. We do not have explicit information about the proportionality constant $a_{2}$ in (25), so we can only determine the ratio $g_{2} / a_{2}$ from this information, $g_{2} / a_{2} \sim\left(D t_{\mathrm{f}}\right)^{1 / 2} / L \sim 0.14$. If $a_{2} \sim a_{1}=4$, this would give $g_{2} \sim 0.6$, but we have no basis for this particular choice. However, since we assume that both $a_{d}$ and $g_{d}$ are independent of $L$, we can use the ratio obtained above to predict the crossover times $t_{\mathrm{f}}$ for the other lattices according to the relation $t_{\mathrm{f}} \sim 0.041 L^{2}$. We thus find for $L=200$ that $t_{\mathrm{f}} \sim 1660$, for $L=600$ that $t_{\mathrm{f}} \sim 1.5 \times 10^{4}$, and for $L=2000$ that $t_{\mathrm{f}} \sim 1.7 \times 10^{5}$. These are all consistent with the simulation results. Again, to emphasize this point, in fig. 6 we have replotted the results of fig. 5 as a function of the scaled time $t / L^{2}$. On this scale the finite size effects for all the finite lattices begin to appear at ap-

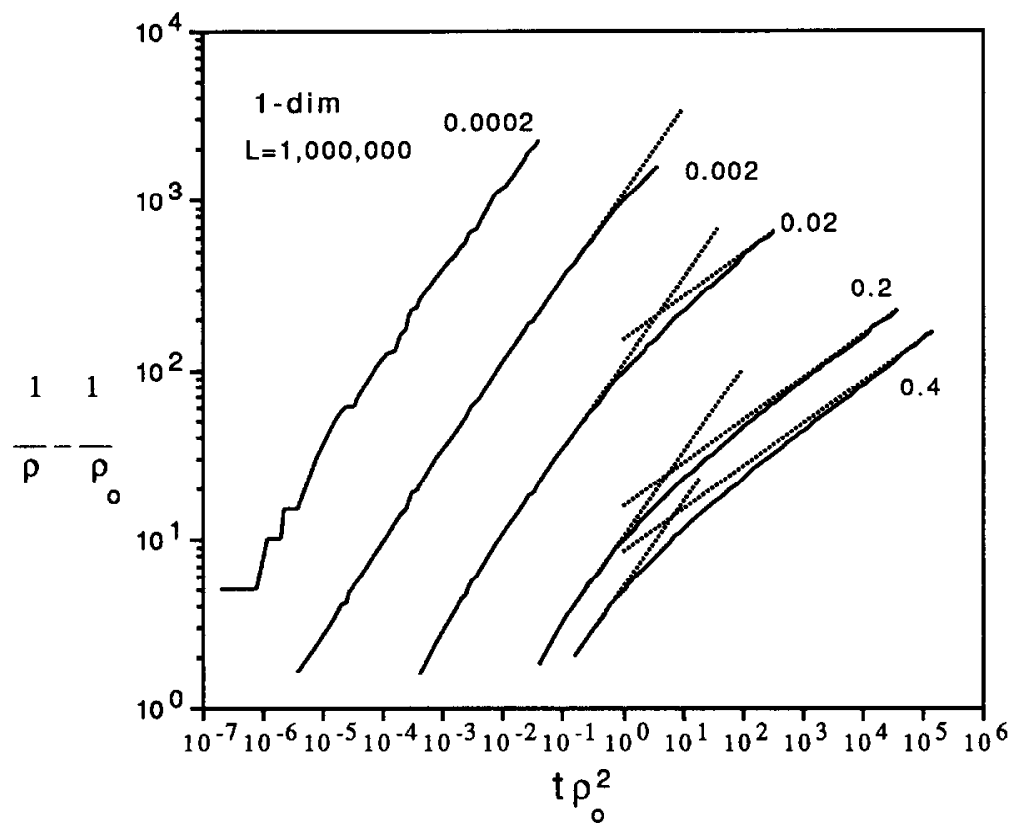

Fig. 4. $\langle\rho\rangle^{-1}-\rho_{0}^{-1}$ versus $t \rho_{0}^{2}$ in one dimension with a random initial condition for the various initial densities indicated. Simulations are on one-dimensional lattices of one million sites. The initial densities are in number per lattice site. 


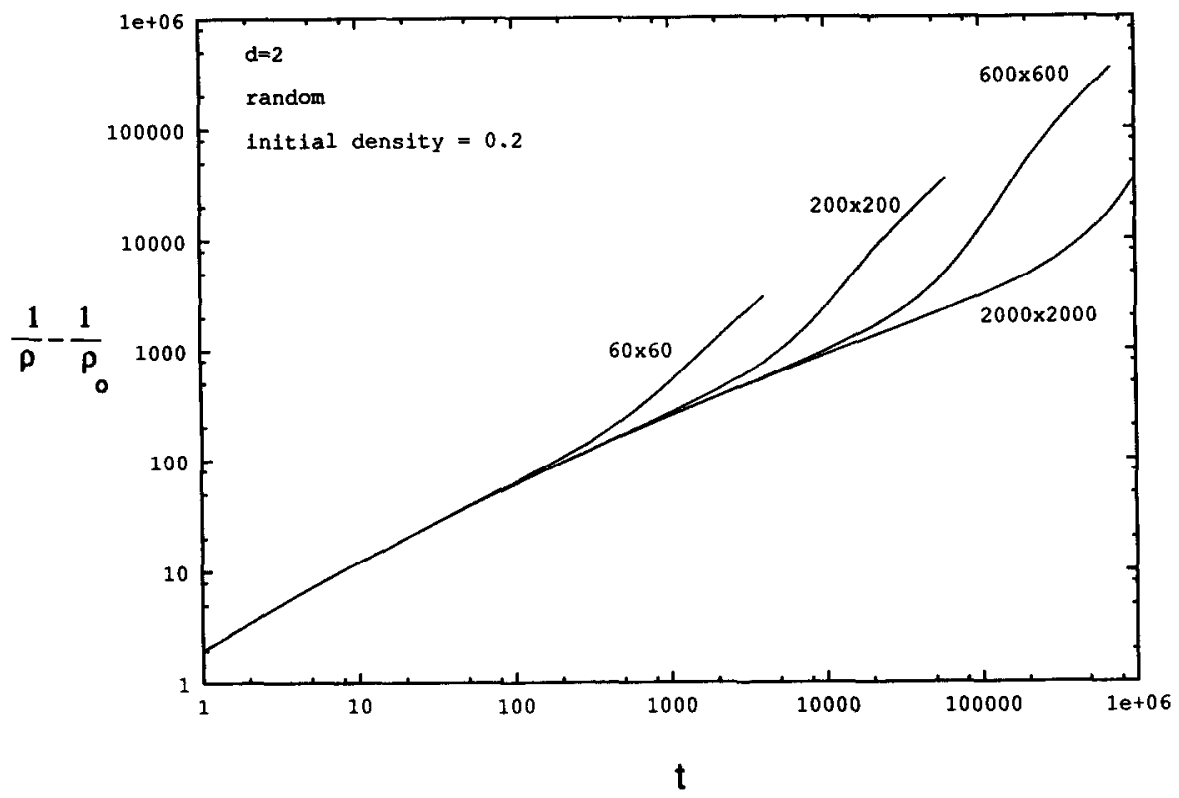

Fig. 5. $\langle\rho\rangle^{-1}-\rho_{0}^{-1}$ versus $t$ for two-dimensional lattices of various sizes $L \times L$ with a random initial condition. The initial density of each species is $\rho_{0}=0.2$ in all cases.

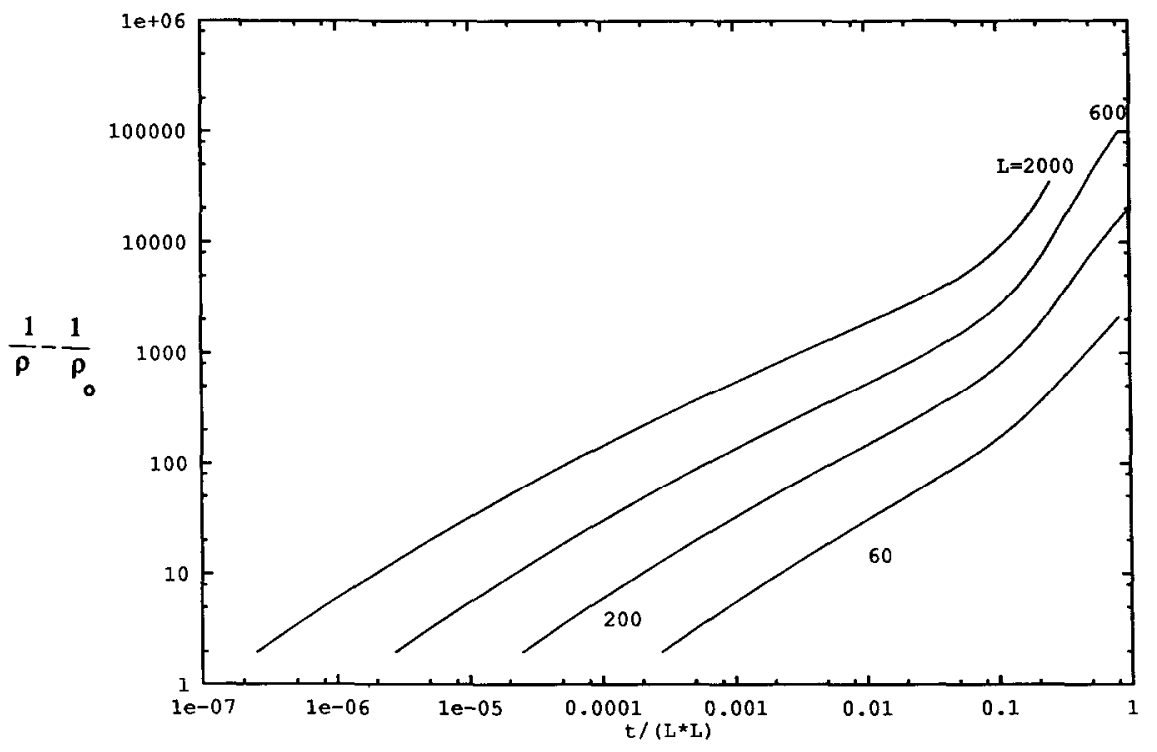

Fig. 6. $\langle\rho\rangle^{-1}-\rho_{0}^{-1}$ versus rescaled time $t / L^{2}$ for the same cases as in fig. 5 .

proximately $t_{\mathrm{f}} / L^{2} \sim 4 \times 10^{-2}$. This figure again makes evident the scaling relation $t_{\mathrm{f}} \sim L^{2}$.

Fig. 7 shows our Monte Carlo results for the reactant density as a function of scaled time in a two-di- mensional lattice for various initial densities $\rho_{0}$. The crossover from the early time $t^{-1} \mathrm{~A}+\mathrm{A}$ behavior to the $t^{-1 / 2}$ Zeldovich behavior has been noted at the higher initial densities (we have not explicitly drawn 


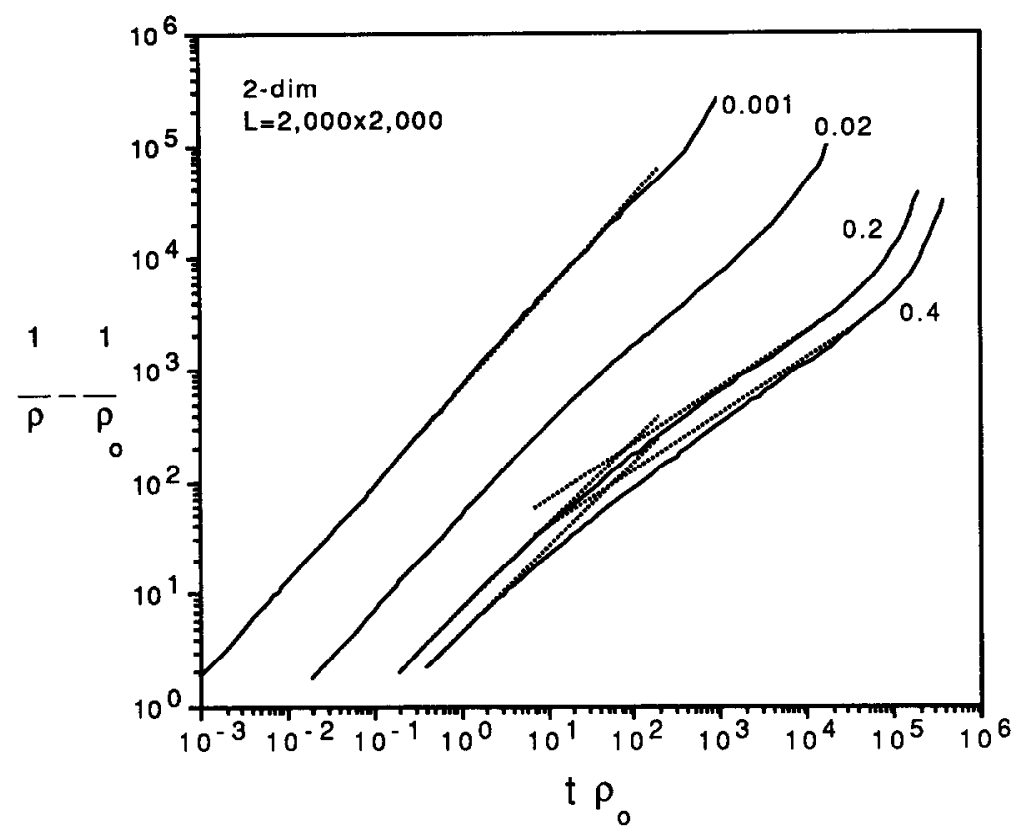

Fig. 7. $\langle\rho\rangle^{-1}-\rho_{0}^{-1}$ versus $t \rho_{0}$ in two dimensions with a random initial condition for the various initial densities indicated. Simulations are on two-dimensional lattices of $2000 \times 2000$ sites.

in the two slope lines for $\rho_{0}=0.02$ although the transition is visible there as well; at the lowest density finite size effects set in too soon to see this transition ). Again the insensitivity of $t_{\mathrm{s}} \rho_{0}$ to the initial density as predicted in (24) is evident. With the value $f_{d} \sim 0.03$ read from these results, one can see that the prediction $\rho_{\mathrm{s}} \sim f_{d} \rho_{0}$ is again excellent (i.e., $f_{d}$ is independent of $\rho_{0}$ ). At very long times the crossover to finite size effects is apparent in the upturn of all the curves.

\section{Conclusions}

In this section we summarize the highlights of our findings, following the kinetic progressions shown in fig. 1. We predict the following sequence of behaviors. First at early times the $A+B$ reaction behaves the same as the $\mathrm{A}+\mathrm{A}$ reaction. Both exhibit classical behavior at very early times but quickly cross over to the depletion zone behavior $t^{-d / 2}$ for $d \leqslant 2$ with logarithmic corrections for $d=2$ and classical behavior for $d \geqslant 3$. This parallel behavior continues up to a time $t_{\mathrm{s}}$, when segregation effects begin in the A+B reac- tion. We estimate the time $t_{\mathrm{s}}$ in eq. (24) and, in particular, predict the product $t_{\mathrm{s}} \rho_{0}^{2 / d}$ to be independent of the initial density $\rho_{0}$. The transition to the Zeldovich regime is therefore expected at shorter times for higher initial densities. We also predict the crossover density $\rho_{\mathrm{s}}$ to be proportional to $\rho_{0}$. Our simulations in both one and two dimensions confirm these predictions quantitatively. Thus, a simulation for a single initial density in a particular dimension allows us to predict this crossover quantitatively for any other initial density. We find that in one dimension $t_{\mathrm{s}} \rho_{0} \sim \mathrm{O}\left(10^{0}\right)-\mathrm{O}\left(10^{1}\right)$ and $\rho_{\mathrm{s}} / \rho_{0} \sim 0.3-0.5$. In two dimensions $t_{\mathrm{s}} \rho_{0} \sim \mathrm{O}\left(10^{1}\right)-\mathrm{O}\left(10^{2}\right)$ and $\rho_{\mathrm{s}} / \rho_{0} \sim 0.03$. From these results we can also predict whether the transition to the Zeldovich regime occurs earlier or later in two dimensions than in one dimension for a given value of $\rho_{0}$. We find that $t_{\mathrm{s}}(2$ dimensions $) / t_{\mathrm{s}}(1$ dimension $)=K \rho_{0}$ where $K$ is $\mathrm{O}\left(10^{1}\right)-\mathrm{O}\left(10^{2}\right)$. Thus, except for very low initial densities, the transition will set in later in two dimensions.

The Zeldovich regime in all cases ends when finite lattice size effects set in. These effects begin when the average linear size of each kind of aggregate (which grows in time as $t^{1 / 2}$ ) is within an order of magnitude 
of the size of the system, independently of the size of the system. In other words, the finite size effects for lattices of linear size $L$ start at a time $t_{\mathrm{f}} \sim L^{2}$. Furthermore, from the finite size results we obtain information about the segregation process in infinite lattices.

We can thus summarize the major novel features of our results as follows:

(1) At early times the $A+B \rightarrow 0$ system does not segregate. Instead it behaves exactly as does the $\mathrm{A}+\mathrm{A} \rightarrow 0$ system, with both classical $t^{-1}$ and (for $d \leqslant 2$ ) nonclassical $t^{-d / 2}$ behavior.

(2) This behavior stops at a time $t_{\mathrm{s}}$, when the segregation process begins. We have found scaling laws for $t_{\mathrm{s}}$ and for the density $\rho_{\mathrm{s}}$ at time $t_{\mathrm{s}}$. Our simulations yield the unknown dimension-specific parameters for $d=1$ and $d=2$.

(3) Eventually finite system size effects set in when the segregated aggregates are within an order of magnitude of the size of the system (linear dimension) at a time $t_{\mathrm{f}}$ whose scaling behavior we have confirmed. Again, we have determined dimension-specific parameters for $d=1$ and $d=2$ from our simulations. These results provide information on the segregation process in infinite lattices.

Finally, our simulations are in excellent agreement with our theoretical predictions.

\section{Acknowledgement}

We gratefully acknowledge support from the US Department of Energy Grant No. DE-FG0386ER13606 (KL), from the National Science Foundation Grant No. DMR-911622 (RK), and from NATO grant No. CRG 920029 (PA).

\section{Appendix A. Solution of difference equations}

The solution of the linear diffusion equation (4) is well known to be given by

$\gamma(\boldsymbol{r}, t)=\frac{1}{V} \sum_{\boldsymbol{k}} \int \mathrm{d} \boldsymbol{r}^{\prime} \exp \left[-\mathrm{i} \boldsymbol{k} \cdot\left(\boldsymbol{r}-\boldsymbol{r}^{\prime}\right)\right] \exp \left(-D k^{2} t\right) \gamma\left(\boldsymbol{r}^{\prime}, 0\right)$,

where (for periodic boundary conditions) $k=2 \pi n / V$ and $n$ is a $d$-tuple of integers.

We need to evaluate

$$
\begin{aligned}
& \left\langle\gamma^{2}(r, t)\right\rangle=\frac{1}{V^{2}} \sum_{k_{1}} \sum_{k_{2}} \int \mathrm{d} r_{1} \exp \left[-\mathrm{i} k_{1} \cdot\left(r-r_{1}\right)\right] \int \mathrm{d} r_{2} \exp \left[-\mathrm{i} k_{2} \cdot\left(r-r_{2}\right)\right] \\
& \quad \times \exp \left[-D\left(k_{1}^{2}+k_{2}^{2}\right) t\right]\left\langle\gamma\left(\boldsymbol{r}_{1}, 0\right) \gamma\left(\boldsymbol{r}_{2}, 0\right)\right\rangle,
\end{aligned}
$$

where the angular brackets indicate the average over the initial distribution of A's and B's. The initial difference variable is given by half the difference between the two expressions in (6), so that

$$
\left\langle\gamma\left(\boldsymbol{r}_{1}, 0\right) \gamma\left(\boldsymbol{r}_{2}, 0\right)\right\rangle=\frac{1}{4}\left\langle\sum_{j=1}^{N}\left[\delta\left(\boldsymbol{r}_{1}-\boldsymbol{r}_{j}^{\mathrm{A}}\right)-\delta\left(\boldsymbol{r}_{1}-\boldsymbol{r}_{j}^{\mathrm{B}}\right)\right] \sum_{j=1}^{N}\left[\delta\left(\boldsymbol{r}_{2}-\boldsymbol{r}_{j}^{\mathrm{A}}\right)-\delta\left(\boldsymbol{r}_{2}-\boldsymbol{r}_{j}^{\mathrm{B}}\right)\right]\right\rangle .
$$

For random initial conditions this expression can immediately be rewritten as

$$
\begin{aligned}
& \left\langle\gamma\left(\boldsymbol{r}_{1}, 0\right) \gamma\left(\boldsymbol{r}_{2}, 0\right)\right\rangle=-\frac{1}{4} N^{2}\left\langle\delta\left(\boldsymbol{r}_{1}-\boldsymbol{r}_{1}^{\mathrm{A}}\right) \delta\left(\boldsymbol{r}_{2}-\boldsymbol{r}_{1}^{\mathrm{B}}\right)\right\rangle-\frac{1}{4} N^{2}\left\langle\delta\left(\boldsymbol{r}_{1}-\boldsymbol{r}_{1}^{\mathrm{B}}\right) \delta\left(\boldsymbol{r}_{2}-\boldsymbol{r}_{1}^{\mathrm{A}}\right)\right\rangle \\
& \quad+\frac{1}{4} N(N-1)\left\langle\delta\left(\boldsymbol{r}_{1}-\boldsymbol{r}_{1}^{\mathrm{A}}\right) \delta\left(\boldsymbol{r}_{2}-\boldsymbol{r}_{2}^{\mathrm{A}}\right)\right\rangle+\frac{1}{4} N(N-1)\left\langle\delta\left(\boldsymbol{r}_{1}-\boldsymbol{r}_{1}^{\mathrm{B}}\right) \delta\left(\boldsymbol{r}_{2}-\boldsymbol{r}_{2}^{\mathrm{B}}\right)\right\rangle \\
& \quad+\frac{1}{4} N\left\langle\delta\left(\boldsymbol{r}_{1}-\boldsymbol{r}_{1}^{\mathrm{A}}\right) \delta\left(\boldsymbol{r}_{2}-\boldsymbol{r}_{1}^{\mathrm{A}}\right)\right\rangle+\frac{1}{4} N\left\langle\delta\left(\boldsymbol{r}_{1}-\boldsymbol{r}_{1}^{\mathrm{B}}\right) \delta\left(\boldsymbol{r}_{2}-\boldsymbol{r}_{1}^{\mathrm{B}}\right)\right\rangle,
\end{aligned}
$$

where we have collected and properly weighted (by their frequency of occurrence) the different types of terms that occur in (A.3). In all but the last two terms in (A.4) the random initial distribution (7) allows us to write the averages of products as products of averages, e.g., 


$$
\begin{gathered}
\left\langle\delta\left(r_{1}-r_{1}^{\mathrm{A}}\right) \delta\left(r_{2}-r_{1}^{\mathrm{b}}\right)\right\rangle=\left\langle\delta\left(r_{1}-r_{1}^{\mathrm{A}}\right)\right\rangle\left\langle\delta\left(r_{2}-r_{1}^{\mathrm{B}}\right)\right\rangle \\
=\int \mathrm{d} r_{1}^{\mathrm{A}} \frac{1}{V} \delta\left(r_{1}-r_{1}^{\mathrm{A}}\right) \int \mathrm{d} r_{1}^{\mathrm{B}} \frac{1}{V} \delta\left(r_{2}-r_{1}^{\mathrm{B}}\right)=\frac{1}{V^{2}} .
\end{gathered}
$$

The last two terms of (A.4) contain an average of the form

$$
\left\langle\delta\left(r_{1}-r_{1}^{\mathrm{A}}\right) \delta\left(r_{2}-r_{1}^{\mathrm{A}}\right)\right\rangle=\int \mathrm{d} r_{1}^{\mathrm{A}} \frac{1}{V} \delta\left(r_{1}-r_{1}^{\mathrm{A}}\right) \delta\left(r_{2}-r_{1}^{\mathrm{A}}\right)=\frac{1}{V} \delta\left(r_{1}-r_{2}\right) .
$$

Thus eq. (A.4) can be rewritten as

$$
\left\langle\gamma\left(\boldsymbol{r}_{1}, 0\right) \gamma\left(\boldsymbol{r}_{2}, 0\right)\right\rangle=-\frac{N}{2 V^{2}}+\frac{N}{2 V} \delta\left(\boldsymbol{r}_{1}-\boldsymbol{r}_{2}\right) .
$$

Substituting this result into (A.2) leaves

$$
\begin{aligned}
& \left\langle\gamma^{2}(r, t)\right\rangle=-\frac{N}{2 V^{4}}\left(\sum_{k} \int \mathrm{d} r_{1} \exp \left[-\mathrm{i} k \cdot\left(r-r_{1}\right)\right] \exp \left(-D k^{2} t\right)\right)^{2} \\
& +\frac{N}{2 V^{3}} \sum_{k_{1}} \sum_{k_{2}} \int \mathrm{d} r_{1} \exp \left[-\mathrm{i}\left(k_{1}+k_{2}\right) \cdot\left(r-r_{1}\right)\right] \exp \left[-D\left(k_{1}^{2}+k_{2}^{2}\right) t\right] .
\end{aligned}
$$

The first integral-sum in (A.8) gives

$\sum_{\boldsymbol{k}} \int \mathrm{d} \boldsymbol{r}_{1} \exp \left[-\mathrm{i} k \cdot\left(r-r_{1}\right)\right] \exp \left(-D k^{2} t\right)=\sum_{k} V \delta_{k, 0} \exp (-D k t)=V$.

The second integral-sum in (A.8) gives

$\sum_{k_{1}} \sum_{k_{2}} \int \mathrm{d} \boldsymbol{r}_{1} \exp \left[-\mathrm{i}\left(\boldsymbol{k}_{1}+\boldsymbol{k}_{2}\right) \cdot\left(\boldsymbol{r}-\boldsymbol{r}_{1}\right)\right] \exp \left[-D\left(k_{1}^{2}+k_{2}^{2}\right) t\right]=V \sum_{\boldsymbol{k}_{1}} \exp \left(-2 D k_{1}^{2} t\right)$

Collecting (A.9) and (A.10) in (A.8) gives us so far

$\left\langle\gamma^{2}(r, t)\right\rangle=-\frac{N}{2 V^{2}}+\frac{N}{2 V^{2}} \sum_{k_{1}} \exp \left(-2 D k_{1}^{2} t\right)$.

The sum in (A.11) cannot in general be done in closed form. However, it can be carried out in the largevolume limit, where the sum can be converted to an integral:

$$
\lim _{V \rightarrow \infty} \frac{1}{V} \sum_{k_{1}} \exp \left(-2 D k_{1}^{2} t\right)=\frac{1}{(2 \pi)^{d}} \int \mathrm{d} k \exp \left(-2 D k^{2} t\right)=\frac{1}{(2 \pi)^{d}} \frac{2 \pi^{d / 2}}{\Gamma(d / 2)} \int_{0}^{\infty} \mathrm{d} k k^{d-1} \exp \left(-2 D k^{2} t\right) .
$$

Clearly the first term in (A.11) is negligible in this limit and so we rewrite (A.11) as

$$
\left\langle\gamma^{2}(r, t)\right\rangle=\frac{N}{V} \frac{1}{2^{d} \pi^{d / 2} \Gamma(d / 2)} \int_{0}^{\infty} \mathrm{d} k k^{d-1} \exp \left(-2 D k^{2} t\right)=\frac{N}{V} \frac{1}{2(8 \pi D t)^{d / 2}} .
$$

\section{Appendix B. Initial averages}

Several $t=0$ averages appear in various contexts in the discussion in sections 2 and 4 . It is useful to first 
present these averages for a discrete system in which the A and B molecules occupy lattice sites separated by a lattice constant $a$. Subsequently we discuss the continuum limit of these results. In all the derivations in this appendix we retain the superscript "lat" on the lattice densities in order to avoid confusion.

Molecules A and B are initially located at sites $l_{j}^{\mathrm{A}}$ and $l_{j}^{\mathrm{B}}$, respectively, with $j=1,2, \ldots, N$. The random initial distribution is reflected in the probabilities that replace $p\left(r^{A}\right) \mathrm{d} r^{A}$ and $p\left(r^{B}\right) \mathrm{d} r^{B}$ used in eq. (7), $p\left(l^{\mathrm{A}}\right)=p\left(l^{\mathrm{B}}\right)=1 / L$. The average initial density of A molecules is given by

$$
\left\langle\rho_{\mathrm{A}}^{\mathrm{iat}}(l, 0)\right\rangle=\sum_{\left\{l_{j}^{\mathrm{A}}\right\}} \rho_{\mathrm{A}}^{\mathrm{lat}}(l, 0) \sum_{j=1}^{N} p\left(l_{j}^{\mathrm{A}}\right),
$$

where in analogy with (2.1a) we have

$\rho_{\mathrm{A}}^{\text {lat }}(l, 0)=\sum_{j=1}^{N} \delta\left(l, l_{j}^{\mathrm{A}}\right)$

and $\delta$ represents a Kronecker delta. Substituting (B.2) into (B.1) gives

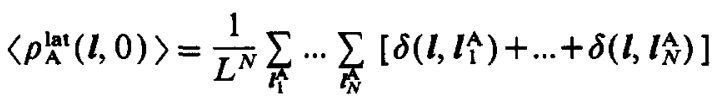

$$
\begin{aligned}
& =\frac{N}{L^{N}} \sum_{\Gamma_{1}^{\lambda}} \cdots \sum_{\lambda_{N}^{\Lambda}} \delta\left(l, l_{1}^{\mathrm{A}}\right)=\frac{N}{L^{N}} L^{N-1} \sum_{\Gamma_{1}^{A}} \delta\left(l, l_{1}^{\mathrm{A}}\right)=\frac{N}{L} .
\end{aligned}
$$

The average of the product of the initial densities of A and B molecules is deduced in exactly the same way and is given by

$\left\langle\rho_{\mathrm{A}}^{\text {lat }}(l, 0) \rho_{\mathrm{B}}^{\text {lat }}(l, 0)\right\rangle=\left\langle\rho_{\mathrm{A}}^{\text {lat }}(l, 0)\right\rangle\left\langle\rho_{\mathrm{B}}^{\text {lat }}(l, 0)\right\rangle=\frac{N^{2}}{L^{2}}$.

The average of the square of the initial density of A particles is

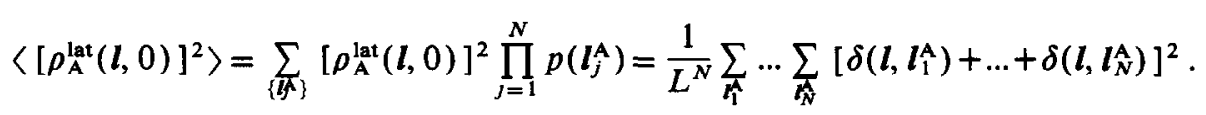

When one carries out the square in the summand of (B.5), two types of terms (with appropriate weights determined by their frequency of occurrence) appear:

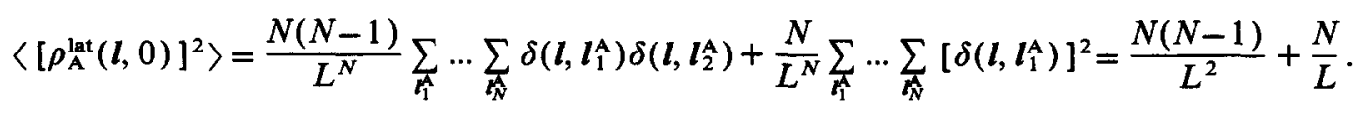

Note that from (B.3) and (B.6) it follows that $\left\langle\left[\rho_{\mathrm{A}}^{\text {lat }}(I, 0)\right]^{2}\right\rangle \neq\left\langle\rho_{\mathrm{A}}^{\text {lat }}(l, 0)\right\rangle^{2}$ and that in fact

$$
\left\langle\left[\rho_{\mathrm{A}}^{\text {lat }}(l, 0)\right]^{2}\right\rangle-\left\langle\rho_{\mathrm{A}}^{\text {lat }}(l, 0)\right\rangle^{2}=\frac{N}{L}-\frac{N}{L^{2}},
$$

that is, the difference (B.7) is larger than $\left\langle\rho_{\mathrm{A}}^{\text {lat }}\right\rangle^{2}$ when $N / L<1$.

The continuum version of averages such as calculated in (B.1) is

$$
\left\langle\rho_{\mathrm{A}}(\boldsymbol{r}, 0)\right\rangle=\int \ldots \int \prod_{j=1}^{N} \mathrm{~d} \boldsymbol{r}_{j}^{\mathrm{A}} \rho_{\mathrm{A}}(r, 0) \prod_{j=1}^{N} p\left(\boldsymbol{r}_{j}^{\mathrm{A}}\right),
$$

where $\rho_{\mathrm{A}}(\boldsymbol{r}, 0)$ is given in (6) and $p\left(\boldsymbol{r}^{\mathrm{A}}\right)$ is given following eq. (7). The calculation of $\left\langle\rho_{\mathrm{A}}(\boldsymbol{r}, 0)\right\rangle$ and of $\left\langle\rho_{\mathrm{A}}(\boldsymbol{r}\right.$, 0) $\rho_{\mathrm{B}}(r, 0)>$ is straightforward and yields precisely what one expects from (B.3) and (B.4):

$$
\left\langle\rho_{\mathrm{A}}(r, 0)\right\rangle=\frac{N}{V}, \quad\left\langle\rho_{\mathrm{A}}(r, 0) \rho_{\mathrm{B}}(r, 0)\right\rangle=\frac{N^{2}}{V^{2}}
$$


The correspondence between the discrete and continuum mean square densities is somewhat more problematic, as can be seen from (B.6). Dividing (B.6) by $a^{2 d}$ yields

$$
\left\langle\rho_{\mathrm{A}}^{2}(r, 0)\right\rangle=\frac{N(N-1)}{V^{2}}+\frac{N}{V} \frac{1}{a^{d}} \text {. }
$$

The second term clearly diverges as $a \rightarrow 0$. Indeed, a calculation based directly on the continuum average yields

$$
\left\langle\rho_{\mathrm{A}}^{2}(\boldsymbol{r}, 0)\right\rangle=\frac{N(N-1)}{V^{2}}+\frac{N}{V} \int \mathrm{d} \boldsymbol{r}^{\mathrm{A}}\left[\delta\left(\boldsymbol{r}-\boldsymbol{r}^{\mathrm{A}}\right)\right]^{2},
$$

where the second term, being an integral of the square of a delta function, clearly diverges. Eq. (B.11) provides insight into the nature of this divergence.

\section{References}

[1] R. Kopelman, Science 241 (1988) 1620.

[2] V. Kuzovkov and E. Kotomin, Rept. Progr. Phys. 51 (1988) 1479 , and references therein.

[3] A.A. Ovchinnikov and Y.G. Zeldovich, Chem. Phys. 28 (1978) 215.

[4] D. Toussaint and F. Wilczek, J. Chem. Phys. 78 (1983) 2642.

[5] K. Kang and S. Redner, Phys. Rev. Letters 52 (1984) 955.

[6] K. Lindenberg, B.J. West and R. Kopelman, in: Noise and chaos in nonlinear dynamical systems, eds. S. Capelin and F. Moss (Cambridge Univ. Press, Cambridge, 1989), and references therein.

[7] E. Clément, L.M. Sander and R. Kopelman, Phys. Rev. A 39 (1989) 6455, 6466.

[8] E. Clément, L.M. Sander and R. Kopelman, Phys. Rev. A 39 (1989) 6472.

[9] W-S. Sheu, K. Lindenberg and R. Kopelman, Phys. Rev. A 42 (1990) 2279.

[10] M. Bramson and J.L. Lebowitz, J. Stat. Phys. 62 (1991) 297.

[11] G. Zumofen, J. Klafter and A. Blumen, Phys. Rev. A 45 (1992) 8977.

[12] L.W. Anacker and R. Kopelman, Phys. Rev. Letters 58 (1987) 289.

[13] Z. Jiang and C. Ebner, Phys. Rev. 41 (1990) 5333.

[14] P. Argyrakis and R. Kopelman, in: Dynamics in small confining systems, cds. J.M. Drake, J. Klafter and R. Kopelman (Materials Research Society, Pittsburgh, 1990) p. 195.

[15] P. Argyrakis and R. Kopelman, Phys. Rev. A 41 (1990) $2114,2121$.

[16] F. Leyvraz and S. Redner, Phys. Rev. Letters 66 (1991) 2168.

[17] S. Kanno, Progr. Theoret. Phys. 79 (1988) 721, 1330.
[18] J-C. Lin, C.R. Doering and D. Ben-Avraham, Chem. Phys. 146 (1990) 355.

[19] C.R. Doering and D. Ben-Avraham, Phys. Rev. A 38 (1988) 3035.

[20] K. Lindenberg, W-S. Sheu and R. Kopelman, J. Stat. Phys. 65 (1991) 1269.

[21] P. Klymko and R. Kopelman, J. Phys. Chem. 86 (1982) $3686 ; 87$ (1983) 4565.

[22] D.C. Torney and H.M. McConnel, J. Phys. Chem. 87 (1983) 1441.

[23] R. Kopelman, J. Stat. Phys. 42 (1986) 185.

[24] L.W. Anacker and R. Kopelman, J. Chem. Phys. 81 (1984) 6402.

[25] L.W. Anacker, R.P. Parson and R. Kopelman, J. Phys. Chem. 89 (1985) 4758.

[26] P. Evesque and J. Duran, J. Chem. Phys. 80 (1984) 3016; P.W. Klymko and R. Kopelman, J. Phys. Chem. 87 (1983) 4565.

[27] R. Kopelman, S.J. Parus and J. Prasad, Chem. Phys. 128 (1988) 209.

[28] S. Chandrasekhar, Rev. Mod. Phys. 15 (1943) 1.

[29] P. Argyrakis and R. Kopelman, J. Phys. Chem. 93 (1989) 225.

[30] K. Lindenberg, P. Argyrakis and R. Kopelman, to be published.

[31] K. Lindenberg, B.J. West and R. Kopelman, Phys. Rev. A 42 (1990) 890.

[32] K. Lindenberg, B.J. West and R. Kopelman, Phys. Rev. Letters 60 (1988) 1777.

[33] P. Argyrakis and R. Kopelman, Phys. Rev. A 45 (1992) 5814.

[34] P. Argyrakis, Comp. Phys. 6 (1992) 525.

[35] R. Schoonover, D. Ben-Avraham, S. Havlin, R. Kopelman and G.H. Weiss, Physica A 171 (1991) 232, and references therein.

[36] D.C. Torney and H.M. McConnel, Proc. Roy. Soc. A 387 (1983) 147.

[37] R. Kopelman and P. Argyrakis, J. Chem. Phys. 72 (1980) 3053.

[38] F. Leyvraz and S. Redner, Phys. Rev. A 46 (1992) 3132. 